\title{
Substituição do feno de “Coastcross” (Cynodon sp.) por casca de soja na alimentação de borregas confinadas
}

\author{
Replacement of coastcross hay by soybean hulls in ewe lambs diets \\ Janice Barreto de Morais ${ }^{\mathrm{I}}$ Ivanete Susin $^{\mathrm{I}}$ Alexandre Vaz Pires ${ }^{\mathrm{I}}$ \\ Clayton Quirino Mendes ${ }^{I}$ Reinaldo Cunha de Oliveira Júnior ${ }^{I I}$
}

\section{RESUMO}

Quarenta e oito borregas da raça Santa Inês (23,1 $\mathrm{kg}$ de peso vivo e 124 dias de idade) foram utilizadas para avaliar os efeitos da substituição do feno de "Coastcross" por casca de soja (CS) no desempenho em confinamento. Os animais foram distribuídos em um delineamento em blocos completos casualizados com quatro tratamentos e seis repetições. As dietas experimentais foram isonitrogenadas e continham a mesma quantidade de fibra em detergente neutro (FDN). A casca de soja foi incluída nas proporções de 0; 12,5; 25 e 37,5\% da matéria seca das dietas correspondendo aos tratamentos experimentais 0CS, 12,5CS, 25CS e 37,5CS, respectivamente. A FDN da CS substituiu 0; 25; 50 e $75 \%$ da $F D N$ do feno. Houve efeito linear crescente $(P<0,01)$ para consumo de matéria seca $\left(0,95 ; 1,02 ; 1,08\right.$ e $\left.1,20 \mathrm{~kg} \mathrm{dia}^{-1}\right)$ e para ganho de peso diário (113; 137; 150 e $\left.187 \mathrm{~g} \mathrm{dia}^{-1}\right)$ e redução linear $(P<0,01)$ na conversão alimentar $(8,55 ; 7,20$;

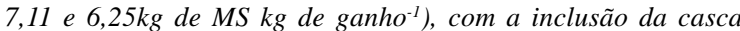
de soja. A casca de soja adicionada em dietas para borregas até $37,5 \%$ da MS melhora o desempenho, fazendo com que os animais alcancem o peso de cobrição mais rápido.

Palavras-chave: fibra em detergente neutro, ovinos, subproduto.

\section{ABSTRACT}

Forty-eight Santa Ines ewe lambs $(23.1 \mathrm{~kg} B W$ and 124 days old) were used to evaluate the replacement of coastcross hay by soybean hulls on feedlot performance. Animals were assigned to a complete randomized block design with four diets and six replicates. All diets were isonitrogenous and contained the same amount of NDF. Soybean hulls replaced hay at $0 \%, 12.5 \%, 25 \%$ or $37.5 \%$ on DM basis, corresponding to the experimental treatments OCS, 12.5CS, 25CS and 37.5CS, respectively. Soybean hulls NDF replaced hay NDF at 0, 25, 50 and $75 \%$. There was a linear increased response $(P<0.01)$ on dry matter intake $\left(0.95,1.02,1.08\right.$ and $\left.1.20 \mathrm{~kg} \mathrm{day}^{-1}\right)$ and average daily gain $\left(113,137,150\right.$ and $\left.187 \mathrm{~g} \mathrm{day}^{-1}\right)$ and a linear improvement $(P<0.01)$ on feed conversion $(8.55,7.20$, 7.11 and $6.25 \mathrm{~kg}$ of $\mathrm{DM} \mathrm{kg}$ of gain $^{-1}$ ) when soybean hulls were added to the diet. When soybean hulls are added to the diet (up to $37.5 \%$, DM basis) there is an improvement on animal performance and the ewe lambs attain breeding weight faster.

Key words: neutral detergent fiber, sheep, byproduct.

\section{INTRODUÇÃO}

O crescente aumento do consumo de carne ovina justifica a necessidade de intensificar a produção de cordeiros, a fim de manter constante o suprimento desse produto no mercado. Para atender a esta demanda, é necessário que o manejo nutricional seja adequado em todas as fases do ciclo de produção. A nutrição inadequada, principalmente durante a fase de crescimento das fêmeas, resulta no atraso da puberdade e, conseqüentemente, da idade à primeira parição.

Sendo assim, é importante atender às exigências do animal em cada etapa do seu desenvolvimento, a fim de se evitar tanto a subnutrição como os ganhos de peso excessivos, que ocasionam maior deposição de gordura na glândula mamária, menor produção de tecido secretor e redução na futura produção de leite (UMBERGER et al., 1985).

Dentre os problemas relacionados à produção de ovinos nos países tropicais estão a baixa

IDepartamento de Zootecnia, Escola Superior de Agricultura Luiz de Queiroz (ESALQ), Universidade de São Paulo (USP), CP 09, 13418-900, Piracicaba, São Paulo, Brasil. E-mail: ivasusin@esalq.usp.br. Autor para correspondência.

IIDepartamento de Zootecnia, Universidade Estadual de Goiás, UnU SLMB/UEG, Anápolis, GO, Brasil. 
qualidade de forragens e a sua sazonalidade de produção, sendo esta última relacionada a períodos prolongados de seca e de umidade, levando a uma produção desigual de forragens ao longo do ano. Além disso, o alto custo para produzir ou adquirir forragens conservadas (silagem e feno) levam à procura de ingredientes mais baratos, de igual ou maior valor nutricional.

Neste contexto, os resíduos ou subprodutos de indústrias alimentícias vêm sendo alvo de estudos na alimentação de ruminantes, sob o ponto de vista econômico e nutricional. A casca de soja, subproduto das indústrias de processamento de soja com alto valor energético, surgiu como uma dessas fontes alternativas de alimento para ruminantes, podendo substituir forragens ou mesmo ingredientes tradicionais, como o milho. Segundo ANDERSON et al. (1988), a casca de soja inteira e pura (livre da contaminação do farelo de soja durante sua obtenção) possui $74 \%$ de FDN na MS, sendo $47 \%$ composta de celulose e $23 \%$ de hemicelulose, o que confere alta digestibilidade da fração fibrosa a este subproduto.

O presente estudo teve por objetivos avaliar a substituição do feno de "Coastcross” (Cynodon spp) por casca de soja na alimentação de borregas Santa Inês confinadas sobre o ganho de peso diário, a conversão alimentar e o consumo de matéria seca.

\section{MATERIAL E MÉTODOS}

Foram utilizadas 48 borregas da raça Santa

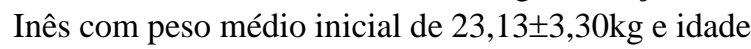
média inicial de $124 \pm 9$ dias. Os animais foram confinados em baias cobertas, com piso de concreto, cocho e bebedouros, sendo dois animais por baia e seis baias por tratamento.

Os animais foram vacinados contra clostridioses antes da desmama (60 dias de idade) e everminados antes do início do experimento. A ocorrência de verminose e coccidiose foi avaliada através de exames quinzenais de fezes para contagem de ovos por grama de fezes (OPG) e controladas quando necessário.

O período experimental teve duração de 84 dias, sendo dividido em três subperíodos de 28 dias de duração. As pesagens foram realizadas nos dias 0; 28; 56 e 84 do período experimental, após jejum alimentar de 16 horas.

As dietas foram isonitrogenadas, sendo que continham a mesma quantidade de FDN e corresponderam aos quatro tratamentos experimentais:
0CS; 12,5CS; 25CS e 37,5CS, ou seja, 0; 12,5; 25 e 37,5\% de inclusão de casca de soja na dieta, respectivamente, de forma que a casca de soja substituiu 0; 25; 50 e 75\% da FDN do feno na dieta. Os teores de FDN e PB (\% MS) obtidos pela análise bromatológica do feno e da casca de soja foram de 72,8 e 11,4\% e de 73,3 e 9,4\%, respectivamente. Para obter a composição química das dietas, os concentrados e o feno foram analisados separadamente. A proporção dos ingredientes e a composição química das dietas estão apresentadas na tabela 1 .

O feno foi processado duas vezes, em uma desintegradora, antes do seu fornecimento aos animais, e o alimento concentrado foi misturado previamente em um misturador horizontal (capacidade de 500kg) e armazenado em tambores plásticos de 200 litros. O feno e o concentrado foram pesados separadamente e misturados nos cochos antes do fornecimento.

As dietas foram fornecidas ad libitum, uma vez ao dia, pela manhã, e as sobras registradas diariamente para a determinação do consumo diário de matéria seca por baia. As sobras foram amostradas semanalmente por baia experimental, conservadas a $-10^{\circ} \mathrm{C}$ e, posteriormente, descongeladas e secadas em estufas de ventilação forçada $\left(55-60^{\circ} \mathrm{C}\right)$, de acordo com GOERING \& VAN SOEST (1970), para a determinação da matéria seca.

As amostras do alimento oferecido e recusado foram moídas em moinhos tipo Wiley, providos de peneiras com crivos de $1 \mathrm{~mm}$, e analisadas para matéria mineral (MM) e MS, de acordo com a ASSOCIATION OF OFFICIAL ANALYTICAL CHEMISTS - AOAC (1990), e FDN, conforme VAN SOEST et al. (1991). Apenas as amostras do alimento oferecido foram analisadas para PB (AOAC, 1990) e FDA segundo GOERING \& VAN SOEST(1970). ONDT das dietas foi estimado conforme LOFGREEN (1953) e CRAMPTON et al. (1957). O cálculo da EM foi baseado nas equações publicadas pelo NRC (1985), em que ED=NDT x 0,04409 e EM é 82\% da ED.

Para estimar a FDN fisicamente efetiva (FDNfe) das dietas oferecidas, foi adotado o método proposto por MERTENS (1997). Aproximadamente 170 a $220 \mathrm{~g}$ de concentrado (de cada tratamento) e $50 \mathrm{~g}$ de feno foram colocados separadamente em uma peneira de $1,18 \mathrm{~mm}$, que foi agitada durante um minuto em um agitador vertical (Cenco-Meinzer Sieve Shaker). Após a agitação, a quantidade de cada amostra retida na peneira foi pesada para a determinação do fator de efetividade física (fef), que é calculado através da proporção de partículas retidas em peneiras de 1,18mm. A concentração de FDN de cada concentrado e do 
Tabela 1 - Proporção dos ingredientes e composição química das dietas experimentais (\% MS).

\begin{tabular}{|c|c|c|c|c|}
\hline & \multicolumn{4}{|c|}{ Tratamentos } \\
\hline & 0CS & $12,5 \mathrm{CS}$ & $25 \mathrm{CS}$ & $37,5 \mathrm{CS}$ \\
\hline \multicolumn{5}{|l|}{ Ingredientes } \\
\hline Feno de “Coastcross" & 50,1 & 37,6 & 25,0 & 12,5 \\
\hline Casca de soja & - & 12,6 & 25,4 & 38,0 \\
\hline Milho & 34,6 & 33,8 & 32,9 & 32,1 \\
\hline Farelo de soja & 13,2 & 14,0 & 14,7 & 15,4 \\
\hline Mistura mineral & 2,1 & 2,0 & 2,0 & 2,0 \\
\hline \multicolumn{5}{|l|}{ Composição química } \\
\hline Proteína bruta & 15,7 & 16,2 & 15,9 & 15,1 \\
\hline Matéria mineral & 6,5 & 6,3 & 5,5 & 5,4 \\
\hline Fibra em detergente ácido & 20,5 & 22,3 & 23,3 & 27,0 \\
\hline Fibra em detergente neutro & 42,7 & 43,8 & 43,1 & 44,0 \\
\hline FDNf & 36,5 & 27,4 & 18,2 & 9,1 \\
\hline FDNcs & - & 9,2 & 18,6 & 27,8 \\
\hline FDNf (\%FDN total) & 85,5 & 62,6 & 42,2 & 20,7 \\
\hline Hemicelulose & 22,2 & 21,5 & 19,8 & 17,0 \\
\hline NDT estimado & 73,7 & 77,2 & 78,2 & 78,4 \\
\hline EM (Mcal.kg MS ${ }^{-1}$ ) & 2,67 & 2,79 & 2,83 & 2,84 \\
\hline
\end{tabular}

Tratamentos: 0CS, 12,5CS, 25CS e 37,5CS = 0\%, 12,5\%, 25\% e 37,5\% de inclusão de casca de soja, respectivamente; FDNf: fibra detergente neutro oriunda da forragem; FDNcs: fibra detergente neutro oriunda da casca de soja; FDNf (\%FDN total): \% da FDN da forragem em relação à FDN total da dieta; NDT: Nutrientes digestíveis totais estimados de acordo com CRAMPTON et al. (1957) e LOFGREEN (1953); EM: Energia metabolizável (Energia Digestível=NDT x 0,04409 e EM = 82\% da ED) (NRC, 1985).

feno foi multiplicada pela fração retida na peneira de $1,18 \mathrm{~mm}$ para calcular a FDNfe dos concentrados e do feno. Os teores de FDNfe das dietas totais foram obtidos através da soma da FDNfe de cada concentrado e da FDNfe do feno (Tabela 2).

O delineamento experimental foi o de blocos completos casualizados com quatro tratamentos e seis repetições, sendo os blocos definidos pelo peso e pela idade iniciais dos animais. As variáveis analisadas foram: ganho de peso diário, consumo de matéria seca e conversão alimentar. Os dados foram analisados pelo procedimento MIXED do pacote estatístico SAS (1996). Os dados foram submetidos à análise de variância e, para as variáveis que obtiveram respostas significativas, utilizou-se o teste de polinômios ortogonais $(\mathrm{P}<0,05)$. Foi utilizado o seguinte modelo estatístico: $\mathrm{Y}_{\mathrm{ijk}}=\mathrm{M}+\mathrm{B}_{\mathrm{i}}+\mathrm{T}_{\mathrm{j}}+\mathrm{P}_{\mathrm{k}}+\mathrm{P}_{\mathrm{k}} \mathrm{xT}_{\mathrm{j}}+\mathrm{E}_{\mathrm{ijk}}$

Em que: $\mathrm{M}=$ Média geral; $\mathrm{B}_{\mathrm{i}}=$ Efeito do bloco; $\mathrm{T}_{\mathrm{j}}=$ Efeito do tratamento; $\mathrm{P}_{\mathrm{k}}=$ Efeito de período; $\mathrm{P}_{\mathrm{k}} \times \mathrm{T}_{\mathrm{j}}=$ Interação entre período e tratamento e $\mathrm{E}_{\mathrm{ijk}}=$ Erro aleatório.

O "Efeito de período" demonstrado no modelo estatístico equivale aos três subperíodos de 28 dias cada, nos quais ocorreram as pesagens dos animais. Este modelo foi utilizado na análise das variáveis: ganho de peso e conversão alimentar. Para a análise do consumo de MS, utilizou-se “semana” no lugar de “período”. O efeito de blocos foi considerado aleatório.

Tabela 2 - Teor de FDNfe das dietas experimentais considerandose a FDNfe do feno e a FDNfe do concentrado.

\begin{tabular}{lccc}
\hline & $\begin{array}{c}\text { \% retida na peneira de } \\
1,18 \mathrm{~mm}\end{array}$ & \%FDN & \%FDNfe \\
\hline Concentrado & & & \\
0CS & 59,4 & 12,5 & 7,4 \\
12,5CS & 52,5 & 26,3 & 13,8 \\
25CS & 58,9 & 33,2 & 19,5 \\
37,5CS & 52,0 & 39,9 & 20,7 \\
Volumoso & & & \\
Feno & 86,3 & 72,8 & 62,8 \\
Dietas totais & & & \\
0CS & - & 42,7 & 35,1 \\
12,5CS & - & 43,8 & 32,2 \\
25CS & - & 43,1 & 30,3 \\
37,5CS & - & 44,0 & 26,0 \\
\hline
\end{tabular}

\% FDN: teor de FDN dos concentrados, do feno e das dietas totais; \% FDNfe: FDN fisicamente efetiva foi calculada pela fração retida na peneira de $1,18 \mathrm{~mm}$ x \% de FDN do concentrado (ou feno). A \% FDNfe das dietas foi obtida pela equação: (\% FDNfe do concentrado x proporção do concentrado em cada dieta) + (\% FDNfe do feno x proporção do feno em cada dieta); Concentrados das dietas 0CS, $12,5 \mathrm{CS}, 25 \mathrm{CS}$ e $37,5 \mathrm{CS}$; Dietas totais: $0 \mathrm{CS}, 12,5 \mathrm{CS}, 25 \mathrm{CS}$ e $37,5 \mathrm{CS}=0 \%, 12,5 \%$, $25 \%$ e $37,5 \%$ de inclusão de casca de soja, respectivamente. 


\section{RESULTADOS E DISCUSSÃO}

Os dados de consumo de matéria seca, ganho de peso vivo e conversão alimentar estão apresentados na tabela 3. Verificou-se efeito linear crescente $(\mathrm{P}<0,01)$ para o ganho de peso $(\mathrm{y}=0,0236 \mathrm{x}+$ 0,$\left.088 ; R^{2}=0,41\right)$ e o consumo de matéria seca em kg.dia${ }^{1}\left(y=0,0826 x+0,8577 ; R^{2}=0,48\right)$, em $\% P V(y=0,1544 x+$ 3,$\left.2957 ; \mathrm{R}^{2}=0,19\right)$ e em $\mathrm{g} / \mathrm{kg} \mathrm{PV}^{0,75}(\mathrm{y}=4,3056 \mathrm{x}+74,385$; $\left.\mathrm{R}^{2}=0,40\right)$ e redução linear $(\mathrm{P}<0,01)$ na conversão alimentar ( $\left.y=-0,7012 x+9,0321 ; R^{2}=0,24\right)$ com a inclusão de $0 ; 12,5 ; 25$ e 37,5\% de casca de soja na dieta. Não houve interação período $\mathrm{x}$ tratamento em nenhuma das variáveis analisadas.

Observando a tabela 3, verifica-se que o consumo de matéria seca aumentou com a inclusão de casca de soja na dieta. A melhor digestibilidade da FDN da casca de soja (MORAIS et al., 2006) e o menor tamanho de partícula, quando comparados ao feno de gramínea grosseiramente picado, provavelmente, contribuíram para uma maior taxa de passagem da dieta e, conseqüentemente, para o aumento do consumo de alimento. A redução no teor de FDN fisicamente efetivo (FDNfe) com a inclusão da casca de soja (Tabela 2), também pode ter contribuído para esses resultados, já que a FDNfe tem relação direta com o tamanho de partículas.

A FDN proveniente de forragem (FDNf) é um fator importante na dieta de ruminantes, pois estimula a mastigação e promove a produção de saliva, que neutraliza os ácidos produzidos durante a fermentação no rúmen. Quantidades inadequadas de FDNf na dieta podem levar à redução do $\mathrm{pH}$ ruminal, do consumo de alimento e comprometer o desempenho do animal. As dietas do presente experimento continham um teor elevado de FDN total (ao redor de 44\%), mas a quantidade de FDNf dos tratamentos 25CS e 37,5CS continham apenas 18,2 e 9,1\% FDNf na MS, respectivamente (Tabela 1), o que poderia comprometer o desempenho do animal.

Contudo, os resultados observados na tabela 3 mostraram que a redução da FDN proveniente de forragem grosseiramente picada em até 9\% não afetou a saúde dos animais e ainda aumentou o ganho de peso conforme a adição de uma fonte de fibra de melhor qualidade oriunda da casca de soja.

Comportamento semelhante de consumo foi averiguado por ARAÚJO (2006), que incluiu 0, 25, 51 e $77 \%$ (da MS) de casca de soja em substituição ao feno de "Coastcross” na alimentação de borregos Santa Inês e verificou aumento linear no consumo de MS. BERGE \& DULPHY (1991) constataram aumento de consumo de MS kg de peso metabólico-1 quando a casca de soja foi adicionada em 0, 30, 60 e 90\% na MS da ração em substituição ao feno de gramínea nativa na alimentação de ovinos.

HSU et al. (1987) substituíram 20\% (da MS da dieta) de feno por casca de soja em dietas para cordeiros e observaram um aumento de $18,4 \%$ no consumo de MS. Este valor foi superior aos 13,7\% de aumento observados no presente experimento, quando a casca substituiu $25 \%$ do feno (Tabela 3 ).

Alguns trabalhos realizados com vacas leiteiras, envolvendo a substituição de forragens por fontes de fibra não-forragem, têm gerado resultados variáveis no consumo de alimento. CUNNINGHAM et al. (1993) e HARMISON et al. (1997) notaram redução linear no CMS com a adição de casca de soja na dieta. Outros autores não verificaram diferenças no CMS quando substituíram feno de alfafa e silagem de milho por casca de soja (SARWAR et al., 1991; SARWAR et al., 1992).

WEIDNER \& GRANT (1994), trabalhando com vacas leiteiras, constataram um aumento no consumo de MS apenas quando a casca de soja substituiu 42\% da MS de uma mistura de silagem de

Tabela 3 - Consumo de matéria seca (CMS), ganho de peso vivo (GPV) e conversão alimentar (CA) das borregas no período experimental.

\begin{tabular}{|c|c|c|c|c|c|c|}
\hline \multirow{2}{*}{ Itens } & \multicolumn{4}{|c|}{ Tratamentos } & \multirow{2}{*}{ EPM } & \multirow{2}{*}{$\mathrm{P}$} \\
\hline & 0CS & $12,5 \mathrm{CS}$ & $25 \mathrm{CS}$ & $37,5 \mathrm{CS}$ & & \\
\hline Peso inicial, kg & 23,02 & 22,93 & 23,45 & 23,13 & 0,70 & 0,7957 \\
\hline Peso final ${ }^{*}, \mathrm{~kg}$ & 32,49 & 34,41 & 36,11 & 38,92 & 0,51 & 0,0001 \\
\hline $\mathrm{CMS}^{*}, \mathrm{~kg} \cdot \mathrm{dia}^{-1}$ & 0,95 & 1,02 & 1,08 & 1,20 & 0,03 & 0,0001 \\
\hline$\% \mathrm{PV}$ & 3,48 & 3,59 & 3,70 & 3,96 & 0,10 & 0,0001 \\
\hline $\mathrm{g} / \mathrm{kg} \mathrm{PV}^{0,75}$ & 79,39 & 82,69 & 85,85 & 92,68 & 1,66 & 0,0001 \\
\hline $\mathrm{GPV}^{*}$, g.dia ${ }^{-1}$ & 113 & 137 & 150 & 187 & 6,00 & 0,0001 \\
\hline CA $^{*}$, kg MS.kg de ganho ${ }^{-1}$ & 8,55 & 7,20 & 7,11 & 6,25 & 0,42 & 0,0001 \\
\hline
\end{tabular}

Tratamentos: 0CS, 12,5CS, 25CS e 37,5CS = 0\%, 12,5\%, 25\% e 37,5\% de inclusão de casca de soja na dieta, respectivamente; EPM = Erro Padrão da Média; $\mathrm{P}$ = Probabilidade de haver efeito significativo entre tratamentos; ${ }^{*}$ Efeito linear $(\mathrm{P}<0,01)$.

Ciência Rural, v.37, n.4, jul-ago, 2007. 
milho e feno de alfafa grosseiramente picado. De acordo com os autores, o menor tamanho de partícula da casca de soja foi um dos determinantes para este aumento do consumo. Outros tratamentos que não continham feno ou que continham um teor mais baixo de casca não apresentaram alteração no consumo.

O ganho de peso vivo aumentou linearmente $(\mathrm{P}<0,01)$ com a inclusão de casca de soja na dieta, variando de 113 a $187 \mathrm{~g} \mathrm{dia}^{-1}$ (Tabela 3). Um padrão de desempenho semelhante foi notado por HSU et al. (1987), segundo os quais o ganho de peso dos cordeiros foi de 140 e $220 \mathrm{~g} \mathrm{dia}^{-1}$, para as dietas com 50 e $70 \%$ (na MS) de inclusão de casca de soja, respectivamente.

O maior ganho de peso observado com a inclusão de casca de soja na dieta foi ocasionado, provavelmente, pelo aumento do consumo de energia digestível, o qual proporcionou um maior aporte energético para o animal, que foi refletido no ganho de peso (Tabela 3), à medida que a casca de soja substituiu o feno.

GOMES et al. (2004) também verificaram melhora linear no ganho de peso diário e no consumo de MS de borregas da raça Churra da Terra Quente quando a casca de soja substituiu o feno de trigo entre 0 e $40 \%$ da MS da dieta.

GARCÉS-YÉPES et al. (1997) constataram maior ganho de peso em novilhos em crescimento suplementados com casca de soja $\left(0,95 \mathrm{~kg} \mathrm{dia}^{-1}\right)$ do que com farelo de soja e milho $\left(0,76 \mathrm{~kg} \mathrm{dia}^{-1}\right)$, em dietas à base de feno de gramínea. De acordo com os autores, este resultado poderia estar associado à maior digestibilidade da FDN no trato digestivo total $(\mathrm{P}<0,01)$ da casca de soja em comparação com a mistura de farelo de soja e milho.

A conversão alimentar melhorou linearmente $(\mathrm{P}<0,01)$ com a inclusão de casca de soja na dieta (Tabela 3). Os valores obtidos indicaram que a eficiência alimentar aumentou, à medida que a quantidade de casca de soja foi incluída na dieta, pois o aumento do ganho de peso foi maior do que o aumento do consumo de MS.

HSU et al. (1987) não observaram diferença significativa na conversão alimentar, quando incluíram $70 \%$ (da MS da dieta) de casca de soja ou milho na alimentação de cordeiros, obtendo valores de 8,17 e $7,20 \mathrm{~kg}$ de MS kgde ganho-1, respectivamente. LOERCH et al. (2001) também não detectaram diferença na conversão alimentar de borregos, quando substituíram um terço do farelo de alfafa por casca de soja, mas obtiveram melhores conversões quando comparadas às do presente estudo, provavelmente, devido ao fato de os machos apresentarem maior eficiência que as fêmeas para converter o alimento em ganho de peso.
SUSIN et al. (1995), trabalhando com dieta contendo $10 \%$ de feno de alfafa como único volumoso para borregas confinadas, observaram conversão alimentar de $6,48 \mathrm{~kg}$ de MS $\mathrm{kg}$ de ganho ${ }^{-1}$, valor este bem próximo ao do presente trabalho $(6,25 \mathrm{~kg}$ MS kg de ganho $^{-1}$ ), no tratamento que continha $37,5 \%$ de casca de soja e $12,5 \%$ de feno de "Coastcross".

\section{CONCLUSÕES}

A casca de soja utilizada em até 37,5\% da MS total da dieta para borregas na fase de crescimento melhora o consumo de MS, o ganho de peso e a conversão alimentar.

A substituição de feno por casca de soja em dietas para borregas faz com que os animais atinjam o peso de cobrição mais rápido e, conseqüentemente, entrem na vida reprodutiva mais cedo.

\section{REFERÊNCIAS}

ANDERSON, S.J. et al. Digestibility and utilization of mechanically processed soybean hulls by lambs and steers. Journal of Animal Science, Champaign, v.66, p.2965-2975, 1988.

ARAÚJO, R.C. Produção de leite e atividade ovariana pós-parto de ovelhas Santa Inês alimentadas com casca de soja em substituição ao feno de "coastcross" (Cynodon sp.). 2006. 135f. Dissertação (Mestrado em Ciência Animal e Pastagem) - Curso de Pós-graduação em Ciência Animal e Pastagens, Escola Superior de Agricultura "Luiz de Queiroz", Universidade de São Paulo.

ASSOCIATION OF OFFICIAL ANALYTICAL CHEMISTS. Official Methods of Analysis. 15.ed. Arlington, 1990. V.1, 1117p.

BERGE, P.; DULPHY, J.P. Étude des interactions entre fourrage et aliment concentré chez le mouton. II. Facteurs de variation de la digestibilité. Annales de Zootechnie, v.40, p.227-246, 1991.

CRAMPTON, E.W. et al. The calorie value of TDN. Journal of Animal Science, v.16, p.541-545, 1957.

CUNNINGHAM, K.D. et al. Nutrient digestion, nitrogen and amino acid flows in lactating cows fed soybean hulls in place of forage or concentrate. Journal of Dairy Science, v.76, p.3523-3535, 1993.

GARCÉS-YÉPEZ, P. et al. Effects of supplemental energy source and amount of forage intake and performance by steers and intake and diet digestibility by sheep. Journal of Animal Science, v.75, p.1918-1925, 1997.

GOERING, H.K.; VAN SOEST, P.J. Forage fiber analysis (apparatus, reagents, procedures and some applications). Washington: Agricultural Research Service, 1970. 19p. (Agricultural Handbook, 379).

GOMES, M.J. et al. Response of lambs fed wheat straw-based diets to supplementation with soybean hulls. Australian Journal of Agricultural Research, v.55, p.261-272, 2004. 
HARMISON, B. et al. Effect of percentage of dietary forage neutral detergent fiber and source of starch on performance of lactating Jersey cows. Journal of Dairy Science, v.80, p.905911, 1997.

HSU, J.T. et al. Evaluation of corn fiber, cottonseed hulls, oat hulls and soybean hull as roughage sources for ruminants. Journal of Animal Science, v.65, p.244-255, 1987.

LOERCH, S.C. et al. Utilization of corn and soyhulls in lamb finishing diets. Athens: Ohio State University, Department of Animal Science, 2001. 3p. (Special Circular, n.181).

LOFGREEN, G.P. The estimation of total digestible nutrients from digestible organic matter. Journal of Animal Science, v.12, p.359-365, 1953.

MERTENS, D.R. Creating a system for meeting the fiber requirements of dairy cows. Journal of Dairy Science, v.80, p.1463-1481, 1997.

MORAIS, J.B. et al. Comportamento ingestivo de ovinos e digestibilidade aparente dos nutrientes de dietas contendo casca de soja. Pesquisa Agropecuária Brasileira, v.41, p.11571164, 2006.

NATIONAL RESEARCH COUNCIL. Nutrient requirements of sheep. 6.ed. Washington: National Academy, 1985. 99p.
SARWAR, M. et al. Effects of replacing neutral detergent fiber of forage with soyhulls and corn gluten feed for dairy heifers. Journal of Dairy Science, v.74, p.1006-1017, 1991.

SARWAR, M. et al. Effects of varying forage and concentrate carbohydrates on nutrient digestibilities and milk production by dairy cows. Journal of Dairy Science, v.75, p.15331542, 1992.

SAS INSTITUTE. SAS User's guide: Statistics. Version 6.12. Cary, 1996. 956p.

SUSIN, I. et al. Effects of limit feeding a high-grain diet on puberty and reproductive performance of ewes. Journal of Animal Science, v.73, p.3206-3215, 1995.

UMBERGER, S.H. et al. Effects of accelerated growth during rearing on reproduction and lactation in ewes lambing at 13 to 15 months of age. Theriogenology, v.23, p.555-564, 1985.

VAN SOEST, P.J. et al. Methods for dietary fiber, neutral detergent fiber, and nonstarch polysaccharides in relation to animal nutrition. Journal of Dairy Science, v.74, p.3583-3597, 1991.

WEIDNER, S.J.; GRANT, R.J. Soyhulls as a replacement for forage fiber in diets for lactating dairy cows. Journal of Dairy Science, v.77, p.513-521, 1994. 